\title{
Co-infection of SARS-COV-2 and influenza A virus: a case series and fast review
}

\author{
Xuan Xiang ${ }^{1}$, Zihao Wang ${ }^{1}$, Xinliang $\mathrm{He}^{1}$, Xiaoshan $\mathrm{Wei}^{1}$, Yanling $\mathrm{Ma}^{1}$, Hui $\mathrm{Li}^{1}$, Long \\ Chen $^{1}$, Xiaorong Wang ${ }^{1}$, and qiong zhou ${ }^{1}$ \\ ${ }^{1}$ Huazhong University of Science and Technology Tongji Medical College
}

June 1, 2020

\begin{abstract}
Background: COVID-19 has become a global pandemic. Studies about SARS-CoV-2 co-infection with influenza A virus (IAV) in the influenza season will contribute to the antiviral interventions of co-infected patients. Methods: A cohort of 145 COVID19 patients in Wuhan union hospital were reviewed and we found 2 patients were co-infected with both SARS-CoV-2 and IAV. Then we searched from PubMed, Web of Science and CNKI with combinations of the following key words: "COVID-19, SARS-COV-2, influenza A and co-infection" from January 1 up to May 1, and 6 studies were included in this descriptive analysis. Results: Of the 13 co-infected patients, 2 were from Wuhan union hospital, another 11 were collected from the reports published on PubMed, Web of Science and CNKI. Of the 13 patients, the median age was 50 years (IQR, 40.5-67.5). Among the 13 patients, $7(53.8 \%)$ were severe types, 7 were males and 5 had comorbidities. The most common symptoms among the 13 patients were cough $(100 \%)$, fever $(92.3 \%)$ and dyspnea (76.9\%). 8 patients had lymphocytopenia on admission and all the 13 patients had abnormal radiological changes in their first examination after admission. The median time from symptom onset to hospital admission was 4.5 days (IQR, 2.75-5.5), and the median time of hospital stay was 17 days (IQR,15-20). 7 patients were discharged, and the clinical outcomes of the remaining 6 patients were unclear. Conclusion: Patients with both SARS-COV-2 and IAV infection showed similar changes in symptoms and radiological images with patients infected with SARS-COV-2 only. SARS-COV-2 co-infection with IAV can lead to more severe clinical condition but had similar hospital stay compared with patients infected with SARS-COV-2 only in the fast review.
\end{abstract}

\section{Introduction}

A new acute respiratory disease reported in last winter named coronavirus disease 2019 (COVID-19), was found to be caused by severe acute respiratory syndrome coronavirus 2 (SARS-CoV-2). It was revealed that SARS-COV-2 virus felled within the subgenus Sarbecovirus of the genus Betacoronavirus, with about $79 \%$ identity to SARS-COV and sharing a similar receptor-binding domain structure to that of SARSCOV, despite a little difference in some residues (Lu et al.2020). This virus now has swept the world with a stunning speed and brought tremendous pressure to the medical system. Many studies had descripted the clinical characteristics and radiological changes of COVID-19. The most common symptoms of COVID-19 were fever and cough (Huang et al, 2020; Wang et al, 2020), and the most representative images of computed tomography were peripheral ground-glass opacities (Chung et al, 2020;Zhang et al, 2020).

Influenza A viruses (IAV) belong to the Orthomyxoviridae family and are further divided into subtypes according to the two surface glycoproteins, haemagglutinin and neuraminidase. IAV can cause acute respiratory illnesses which usually occur in winter seasons periodically and often cause severe illnesses and economic loss around the world (Lafond et al, 2016;Thompson, 2004). Depending on the features of host and virus, patients got infected with IAV could present different symptoms, including fever, chills, myalgia and respiratory symptoms such as dry cough, nasal tampon, etc. The typical changes in chest radiology images of IAV patients were ground-glass opacities and consolidation (Collins et al, 2019;Sargin et al, 2018). 
In clinic, co-infection can happen in viral-bacterial and viral-viral infection during the influenza season and cause more severe clinical conditions (Martin-Loeches et al, 2017;McCullers, 2006). We also find some patients were infected with both SARS-COV-2 and IAV in the COVID-19 pandemic (Ding, Lu, Fan, Xia, \& Liu 2020;Khodamoradi, Moghadami, \& Lotfi, 2020;Wu et al, 2020). Due to the scattered cases, we do not have a profile knowledge about the course of those co-infected patients. It deserves more attention to those patients who suffers from co-infection with SARS-CoV-2 and IAV. Herein we reviewed some published studies and described a cohort of patients co-infected with SARS-CoV-2 and IAV in the COVID-19 pandemic, aiming to explore more knowledge about COVID-19.

\section{Materials and methods}

We retrospectively screened out two patients infected with both SARS-COV-2 and IAV in 145 confirmed COVID-19 patients in Wuhan Union Hospital. Then we searched PubMed (National Library of Medicine, Washington, DC), Web of Science and CNKI (China National Knowledge Infrastructure) from January 1 up to May 1, 2020, using a combination of the following key words: COVID-19, SARS-COV-2, influenza A and co-infection. We also reviewed the reference lists of retrieved articles to search for other proper studies. Studies were selected if they met the flowing criteria: clinical studies and case report on SARS-COV-2 and IAV co-infection.

\section{Data extraction}

For each study, the following information was extracted: the author's last name; year; country; number of patents got co-infected with SARS-COV-2 and IAV; study design; selected clinical data (age; gender; comorbidities; date of symptom onset; date of admission to hospital; days in hospital; symptom before admission; lymphocyte count on admission; oxygen saturation on admission; the first radiological images after admission; treatment; clinical events: acute liver injury, acute respiratory distress syndrome, noninvasive ventilation, endotracheal intubation, intensive care unit (ICU); clinical ending: death or discharged). Patients were divided into moderate type (fever, cough and other symptoms are presented with pneumonia on chest CT scans) and severe type (severe type was defined if one of the following conditions was met: a. respiratory distress, respiratory rate [?] 30 per min; b. oxygen saturation on room air at rest [?] 93\%; c. partial pressure of oxygen in arterial blood / fraction of inspired oxygen [?] $300 \mathrm{mmHg}$ ) according to the Diagnosis and Treatment of Protocol of COVID-19 (National Health Commission, 2020).

\section{Date analysis}

Frequency with percentages (\%) was used to describe categorical variables. Continuous variables were described using median and interquartile range (IQR) values. All statistical analyses were performed with GraphPad Prism 8.0.2.

\section{Results}

We collected 12 articles on SARS -COV -2 and IAV co-infection from PubMed, Web of Science and CNKI and excluded 6 studies for those that did not include clinical information of patients. The remaining 6 articles ( 1 was clinical study and the other 5 were case reports) provided us 11 cases with clinical information. Thus our cohort had 13 patients who suffered from both SARS -COV -2 and IAV co-infection in the pandemic.(Azekawa, Namkoong, Mitamura, Kawaoka, \& Saito, 2020;Ding et al. 2020;Khodamoradi et al. 2020; Konala et al. 2020; Sha 2020; Wu et al. 2020) (Table 1).

In this descriptive study, 7 patients $(53.8 \%)$ were severe cases, $7(53.8 \%)$ were males,the median age was 50 years (IQR, 40.5-67.5). 5 patients (38.5\%) had comorbidities such as hypertension, diabetes, kidney tumor, cerebrovascular disease, coronary artery disease, dyslipidemia, hypothyroidism, pituitary microadenoma, congestive heart failure, chronic kidney disease. The most common symptoms before admission were cough $(100 \%)$, fever $(92.3 \%)$, dyspnea $(76.9 \%)$ and myalgia (53.8\%). Other symptoms such as fatigue, anorexia, headache, nasal tampon, expectoration, pharyngalgia, diarrhea, chest pain and chill were also seen in the cohort (Table 2). 
On admission, 8 patients (61.5\%) had decreased lymphocyte count, 6 of 10 patients (60\%) had lower oxygen saturation (SpO2[?]93\%) on ambient air. All the 13 patients (100\%) had abnormal radiological changes in their first examination after admission. The median time from symptom onset to hospital admission was 4.5 days (IQR, 2.75-5.5), and the median time of hospital stay was 17 days (IQR,15-20). Among the patients, 6 of $9(66.7 \%)$ had oxygen inhalation, including 1 received noninvasive ventilation, and 2 patients received endotracheal intubation in the ICU. 8 of 9 patients $(88.9 \%)$ had oseltamivir treatment, and 7 of 8 patients $(87.5 \%)$ had other antiviral treatment except oseltamivir. 8 patients $(100 \%)$ received antibiotic therapy, only 3 of $8(37.5 \%)$ were treated with corticosteroids. 2 patients suffered from acute liver injury and 2 developed into acute respiratory distress syndrome during the clinical course. 7 patients were discharged. The remaining 6 patients' clinical outcome were not available. (Table 3 ).

\section{Discussion}

Viruses co-infection were usually seen in the influenza epidemic and pandemic (Goka, Vallely, Mutton, \& Klapper, 2013;Meskill, Revell, Chandramohan, \& Cruz, 2017). Goka et al found that IAV and influenza B virus co-infection was associated with a significant increase in risk of admission to ICU or death, whereas co-infection with IAV and other viruses significantly increased the risk of admission to a general ward (Goka et al, 2013). In the COVID-19 pandemic, we found some patients were co-infected with SARS-COV-2 and IAV but we did not know much more about the two viruses' co-infection. To better understand the clinical course of SARS-COV-2 and IAV co-infection, we performed a fast review on patients infected with the two viruses.

In this review, we collected 13 patients co-infected with SARS-COV-2 and IAV in China, Japan, Iran and America (Azekawa et al, 2020;Ding et al, 2020;Khodamoradi et al, 2020;Konala et al, 2020;Sha, 2020;Wu et al, 2020). In the 13 patients, the propotion of sever type was $53.8 \%$ (7 cases), which was much higher than that in COVID-19 (Guan et al, 2020), indicating co-infection with SARS-COV-2 and IAV resulting in more severe condition. IAV infection was more common in children and old people (Thompson, 2004), while we only found one child suffering from SARS-COV-2 and IAV co-infection in our cohort (Sha, 2020). Similar to the COVID-19 patients, the patients co-infected with SARS-COV-2 and IAV also manifested symptoms such as fever, cough, dyspnea and myalgia etc. However, in this review, we found 10 of 13 patients (76.9\%) had dyspnea on admission, higher than that reported by Wang and Huang et al (31.2\% and $55 \%$ respectively) (Huang et al, 2020;Wang et al, 2020). This may remind us that COVID-19 patients co-infected with IAV may develop a more severe disease in lower respiratory tract. The median time of hospital stay in this review was 17 days (IQR,15-20), similar to the finding (median, 18 days, IQR, 14-27) reported by Xu et al (Xu et al, 2020). The reason why patients co-infected with SARS-COV-2 and IAV developed more severe clinical condition while had similar hospital stay need further research. All the patients had typical abnormal radiological changes and 7 of 13 patients (53.8\%) had lower lymphocyte count on admission, which could indicate viral infection but could not tell the pathogens relying on these signs.

In this review, we found 8 of 9 patients $(88.9 \%)$ were treated with oseltamivir, a neuraminidase inhibitor recommended to deal with influenza by the European Union and the USA(European Centre For Disease Control, 2017; Centers for Disease Control and Prevention), and 8 of 8 patients received antibiotic therapy to prevent secondary bacterial infection(McCullers \& Bartmess, 2003). But whether oseltamivir has some effect on SARS-COV-2 viral shedding and whether co-infection with SARS-COV-2 and IAV will cause secondary infection need further investigation. Three patients were treated with corticosteroids, while corticosteroids were not usually recommended in the COVID-19 pandemic concerning about the possible side effects of corticosteroids on virus clearance and association with high rates of complications (Arabi et al, 2018;Stockman, Bellamy \& Garner, 2006). 7 patients were cured and discharged from hospital while 6 patients did not have detailed information on clinical course.

We noticed the patients co-infected with SARS-COV-2 and IAV accounted for a small proportion in the COVID-19 pandemic (Kim, Quinn, Pinsky, Shah, \& Brown, 2020;Nowak, Sordillo, Gitman, \& Paniz Mondolfi 2020). This may due to the interference between viruses (Schultz-Cherry, 2015), and SARS-COV-2 took the dominated place in the pandemic. The mechanism of how SARS-COV-2 and IAV interfere each other deserves 
further study.

Our study has some limitations: first and most, though this was a fast review, our samples were extremely small due to the rare reports on SARS-COV-2 and IAV co-infection. Second, we could not collect detail information about each patient because some information was not available and whether the missing information may have some influence on our results was unknown. Third, the reports did not provide SARS-COV-2 viral shedding time, thus whether co-infection with IAV have some influence on SARS-COV-2 viral shedding remains unknown. Fourth, we could not identify which virus was first infected, which may contribute to account for the co-infection course. Finally, no patient got lower respiratory tract samples for detection for SARS-CoV-2 and IAV in this review, so whether the two viruses were both involved in the pathological progression of pulmonary could not be identified. However, this review highlights that we should pay attention to the problem of co-infection of two or more respiratory pathogens. The association between the occurrence of co-infection and substantially higher severity of disease deserves further study, but a rapid and proper diagnostic of wide spectrum of viral respiratory pathogens reveals an accurate picture of the disease and is essential for appropriate therapeutic management and control of infection.

\section{Acknowledgements and Funding}

This work was supported by National Natural Science Foundation of China (No.81973990, No. 81900096, No.81770090) and Fundamental Research Funds for the Central Universities (No.2020kfyXGYJ030).

\section{Ethical statement}

Data collection and analysis of cases were determined by the National Health Commission of the People's Republic of China to be part of a continuing public health outbreak investigation and were thus considered exempt from institutional review board approval.

\section{Declaration of interests:}

All authors declare no competing interests.

\section{Author contribution statement:}

Qiong Zhou conceived the idea, designed and supervised the study, had full access to all data and took responsibility for the integrity of the data. Xuan Xiang and Zi-Hao Wang participated in the design, implementation and drafting of the article; Hui Li, Yanling Ma and Xiaoshan Wei participated in the collection and collation of clinical data; Xinliang He, Long Chen and Xiaorong Wang participated in the analysis and discussion of clinical data.

\section{Data availability statement}

The data that support the findings of this study are available from PubMed (National Library of Medicine, Washington, DC), Web of Science and CNKI (China National Knowledge Infrastructure), collated and presented in the supplementary 1.

\section{References:}

. National Health Commission, $\begin{gathered}\text { State Administration of } \\ \text { nese Medicine. }\end{gathered}$ Diagnosis and $\begin{gathered}\text { Treatment of Protocol onal Chi- } \\ \text { of COVID-19. }\end{gathered}$
http://www.nhc.gov.cn/xcs/zhengcwj/202003/46c9294a7dfe4cef80dc7f5912eb1989.Shtml, 2020- $03-03$.
2020;. (Reprinted.
$\quad$ European Centre For Disease Control. Scientific-advice-neuraminidase-inhibitors-2017.
https://www.ecdc.europa.eu/sites/default/files/documents/Scientific-advice-neuraminidase-inhibitors-
2017., from

. Centers for Disease Control and Prevention. Influenza. https://www.cdc.gov/flu/treatment/whatyoushould.htm. (Reprinted. 
Arabi, Y. M., Mandourah, Y., Al-Hameed, F., Sindi, A. A., Almekhlafi, G. A., Hussein, M. A.,... Fowler, R. A. (2018). Corticosteroid Therapy for Critically Ill Patients with Middle East Respiratory Syndrome.American Journal of Respiratory and Critical Care Medicine , 197(6), 757-767. doi: $10.1164 / \mathrm{rccm} .201706-1172 \mathrm{OC}$

Azekawa, S., Namkoong, H., Mitamura, K., Kawaoka, Y., \& Saito, F. (2020). Co-infection with SARS-CoV-2 and influenza A virus.IDCases, 20, e775. doi: 10.1016/j.idcr.2020.e00775

Chung, M., Bernheim, A., Mei, X., Zhang, N., Huang, M., Zeng, X.,... Shan, H. (2020). CT Imaging Features of 2019 Novel Coronavirus (2019-nCoV). Radiology , 200230. doi: 10.1148/radiol.2020200230

Collins, J. P., Campbell, A. P., Openo, K., Farley, M. M., Cummings, C. N., Kirley, P. D.,... Anderson, E. J. (2019). Clinical Features and Outcomes of Immunocompromised Children Hospitalized With LaboratoryConfirmed Influenza in the United States, 2011-2015. Journal of the Pediatric Infectious Diseases Society , 8(6), 539-549. doi: 10.1093/jpids/piy101

Ding, Q., Lu, P., Fan, Y., Xia, Y., \& Liu, M. (2020). The clinical characteristics of pneumonia patients coinfected with 2019 novel coronavirus and influenza virus in Wuhan, China. Journal of Medical Virology . doi: $10.1002 / \mathrm{jmv} .25781$

Goka, E., Vallely, P., Mutton, K., \& Klapper, P. (2013). Influenza A viruses dual and multiple infections with other respiratory viruses and risk of hospitalisation and mortality. Influenza and Other Respiratory Viruses , 7(6), 1079-1087. doi: 10.1111/irv.12020

Guan, W., Ni, Z., Hu, Y., Liang, W., Ou, C., He, J.,... Zhong, N. (2020). Clinical Characteristics of Coronavirus Disease 2019 in China. [Journal Article; Research Support, Non-U.S. Gov't]. New England Journal of Medicine , 382(18), 1708-1720. doi: 10.1056/NEJMoa2002032

Huang, C., Wang, Y., Li, X., Ren, L., Zhao, J., Hu, Y.,.. Cao, B. (2020). Clinical features of patients infected with 2019 novel coronavirus in Wuhan, China. The Lancet, 395(10223), 497-506. doi: 10.1016/S0140-6736(20)30183-5

Khodamoradi, Z., Moghadami, M., \& Lotfi, M. (2020). Co-infection of Coronavirus Disease 2019 and Influenza A: A Report from Iran.Archives of Iranian Medicine , 23(4), 239-243. doi: 10.34172/aim.2020.04

Kim, D., Quinn, J., Pinsky, B., Shah, N. H., \& Brown, I. (2020). Rates of Co-infection Between SARS-CoV-2 and Other Respiratory Pathogens. [Journal Article]. JAMA . doi: 10.1001/jama.2020.6266

Konala, V. M., Adapa, S., Gayam, V., Naramala, S., Daggubati, S. R., Kammari, C. B.,... Chenna, A. (2020). Co-infection with Influenza A and COVID-19. European Journal of Case Reports in Internal Medicine, 7(5), 1. doi: 10.12890/2020_001656

Lafond, K. E., Nair, H., Rasooly, M. H., Valente, F., Booy, R., Rahman, M.,... Widdowson, M. (2016). Global Role and Burden of Influenza in Pediatric Respiratory Hospitalizations, 1982-2012: A Systematic Analysis. PLOS Medicine, 13(3), e1001977. doi: 10.1371/journal.pmed.1001977

Lu, R., Zhao, X., Li, J., Niu, P., Yang, B., Wu, H.,... Tan, W. (2020). Genomic characterisation and epidemiology of 2019 novel coronavirus: implications for virus origins and receptor binding. The Lancet, 395(10224), 565-574. doi: 10.1016/S0140-6736(20)30251-8

Martin-Loeches, I., J Schultz, M., Vincent, J., Alvarez-Lerma, F., Bos, L. D., Solé-Violán, J.,... Rodriguez, A. (2017). Increased incidence of co-infection in critically ill patients with influenza. Intensive Care Medicine , 43(1), 48-58. doi: 10.1007/s00134-016-4578-y

McCullers, J. A. (2006). Insights into the Interaction between Influenza Virus and Pneumococcus. Clinical Microbiology Reviews , 19(3), 571-582. doi: 10.1128/CMR.00058-05

McCullers, J. A., \& Bartmess, K. C. (2003). Role of Neuraminidase in Lethal Synergism between Influenza Virus and Streptococcus pneumoniae.The Journal of Infectious Diseases , 187(6), 1000-1009. doi: 


\section{$10.1086 / 368163$}

Meskill, S. D., Revell, P. A., Chandramohan, L., \& Cruz, A. T. (2017). Prevalence of co-infection between respiratory syncytial virus and influenza in children. The American Journal of Emergency Medicine, 35(3), 495-498. doi: 10.1016/j.ajem.2016.12.001

Nowak, M. D., Sordillo, E. M., Gitman, M. R., \& Paniz Mondolfi, A. E. (2020). Co-infection in SARS-CoV-2 infected Patients: Where Are Influenza Virus and Rhinovirus/Enterovirus? Journal of Medical Virology . doi: $10.1002 / \mathrm{jmv} .25953$

Sargin, G., Şentürk, T., Ceylan, E., Telli, M., Çildă̆, S.,... Doğan, H. (2018). Clinical, radiological and prognostic features of influenza cases in the influenza epidemic during years 2016-2017. Tuberkuloz ve Toraks , 66(2), 136-143. doi: 10.5578/tt.66122

Schultz-Cherry, S. (2015). Viral Interference: The Case of Influenza Viruses. Journal of Infectious Diseases , 212(11), 1690-1691. doi: 10.1093/infdis/jiv261

Sha, G. (2020). Co-infection of SARS CoV-2 and influenza A in a child: a case report. Henan J Prev Med , 31(05), 323-324

Stockman, L. J., Bellamy, R., \& Garner, P. (2006). SARS: systematic review of treatment effects. [Journal Article; Research Support, Non-U.S. Gov't; Review; Systematic Review]. PLoS Med , 3(9), e343. doi: 10.1371/journal.pmed.0030343

Thompson, W. W. (2004). Influenza-Associated Hospitalizations in the United States. [Journal Article]. JAMA , 292(11), 1333. doi: 10.1001/jama.292.11.1333

Wang, D., Hu, B., Hu, C., Zhu, F., Liu, X., Zhang, J.,.. Peng, Z. (2020). Clinical Characteristics of 138 Hospitalized Patients With 2019 Novel Coronavirus-Infected Pneumonia in Wuhan, China. JAMA, 323(11), 1061. doi: $10.1001 /$ jama.2020.1585

Wu, X., Cai, Y., Huang, X., Yu, X., Zhao, L., Wang, F.,... Zhan, Q. (2020). Co-infection with SARS-CoV-2 and Influenza A Virus in Patient with Pneumonia, China. [Journal Article]. Emerg Infect Dis , 26(6). doi: 10.3201/eid2606.200299

Xu, K., Chen, Y., Yuan, J., Yi, P., Ding, C., Wu, W.,... Li, L. (2020). Factors associated with prolonged viral RNA shedding in patients with COVID-19. [Journal Article]. Clin Infect Dis . doi: 10.1093/cid/ciaa351

Zhang, R., Ouyang, H., Fu, L., Wang, S., Han, J., Huang, K.,.. . Fu, Z. (2020). CT features of SARS-CoV2 pneumonia according to clinical presentation: a retrospective analysis of 120 consecutive patients from Wuhan city. European Radiology . doi: 10.1007/s00330-020-06854-1

Table 1 Study samples included in the review

Table 2 Basic clinical characteristics of the patents in the included studies

Table 3 Clinical course of the patents in the included studies

Supplementary 1 Data information

\section{Hosted file}

Tble1.docx available at https://authorea.com/users/328031/articles/455427-co-infection-ofsars-cov-2-and-influenza-a-virus-a-case-series-and-fast-review

\section{Hosted file}

Table 2.docx available at https://authorea.com/users/328031/articles/455427-co-infection-ofsars-cov-2-and-influenza-a-virus-a-case-series-and-fast-review

\section{Hosted file}


Table 3.docx available at https://authorea.com/users/328031/articles/455427-co-infection-ofsars-cov-2-and-influenza-a-virus-a-case-series-and-fast-review 